\title{
Bladder Cancer Multidisciplinary Clinic (BCMC) Model Influences Disease Assessment and Impacts Treatment Recommendations
}

Leonidas N. Diamantopoulos ${ }^{\mathrm{a}}$, Brian R. Winters ${ }^{\mathrm{b}}$, Petros Grivas ${ }^{\mathrm{a}, \mathrm{e}}$, Steven D. Ngo ${ }^{\mathrm{c}}$, Jing Zeng ${ }^{d}$, Andrew C. Hsieh ${ }^{\mathrm{a}, \mathrm{e}}$, John L. Gore ${ }^{\mathrm{b}}$, Jay J. Liao ${ }^{\mathrm{d}}$, Evan Y. Yu ${ }^{\mathrm{a}, \mathrm{e}}$, George R. Schade ${ }^{\mathrm{b}, \mathrm{e}}$, Sarah P. Psutka ${ }^{b}$, Michael T. Schweizer ${ }^{\mathrm{a}, \mathrm{e}}$, Jean H. Lee ${ }^{\mathrm{f}}$, Manjiri Dighe ${ }^{\mathrm{f}}$, Daniel W. Lin ${ }^{\mathrm{b}, \mathrm{e}}$, Heather H. Cheng ${ }^{\mathrm{a}, \mathrm{e}}$, Joanna Daya ${ }^{\mathrm{c}}$, Maria S. Tretiakova ${ }^{\mathrm{g}}$, Lawrence D. True ${ }^{\mathrm{g}}$, Kenneth J. Russell ${ }^{\mathrm{d}}$, Funda Vakar-Lopez ${ }^{\mathrm{g}}$, Robert B. Montgomery ${ }^{\mathrm{a}, \mathrm{e}}$ and Jonathan L. Wright ${ }^{\mathrm{b}, \mathrm{e}, *}$

${ }^{a}$ Department of Medicine, Division of Oncology, University of Washington School of Medicine, Seattle Cancer Care Alliance, Seattle, WA, USA

${ }^{\mathrm{b}}$ Department of Urology, University of Washington School of Medicine, Seattle, WA, USA

${ }^{\mathrm{c}}$ University of Washington School of Medicine, Seattle, WA, USA

${ }^{\mathrm{d}}$ Department of Radiation Oncology, University of Washington School of Medicine, Seattle, WA, USA

${ }^{\mathrm{e}}$ Fred Hutchinson Cancer Research Center, Seattle, WA, USA

${ }^{\mathrm{f}}$ Department of Radiology, University of Washington School of Medicine, Seattle, WA, USA

${ }^{\mathrm{g}}$ Department of Pathology, University of Washington School of Medicine, Seattle, WA, USA

\begin{abstract}
.
BACKGROUND: Despite established guidelines for bladder cancer (BC) management, significant treatment variability remains across the United States.

OBJECTIVE: To report the impact of a tertiary center, bladder cancer multidisciplinary clinic (BCMC) on diagnostic evaluation and treatment recommendations in externally referred patients with $\mathrm{BC}$.

METHODS: Our BCMC clinic format includes simultaneous consultation with urologic, medical and radiation oncology, with real-time expert genitourinary pathology and radiology review. We retrospectively assessed all external referrals for concordance between outside radiology/pathology records and BCMC interpretation after central review and explored potential differences between outside treatment plan and BCMC recommendations.

RESULTS: 233 patients with BC were referred to BCMC between the years 2014-2017. Complete radiographic and pathology data were available for 201 patients. Median age was 69 (Interquartile Range: 60-75) and 83\% were performance status ECOG 0-1. After BCMC review of outside records, imaging interpretation was changed in 53 (26\%) patients; pathology was changed in 59 (29\%). Further diagnostic work-up was recommended in $85(42 \%)$ patients. Overall, $56(28 \%)$ patients had a change in their clinical staging. BCMC recommended treatment modification in $117(58 \%)$ patients. Subsequent treatment plan was concordant with BCMC recommendations $91.5 \%$ of the time.
\end{abstract}

\footnotetext{
${ }^{*}$ Correspondence to: Jonathan L. Wright, MD, MS, FACS, Associate Professor of Urology, University of Washington School of Medicine, 1959 NE Pacific Box 356510, Seattle, WA 98195, USA. Tel.: +1 206543 4740; Fax: +1 206543 3272; E-mail: jlwright@uw.edu.
} 
CONCLUSIONS: BCMC resulted in critical radiology and/or pathology diagnostic changes in several cases, with frequent treatment modifications, underscoring the importance of multidisciplinary care in $\mathrm{BC}$ and the considerable contribution of real-time expert pathology and radiology review in diagnostic accuracy. Further studies are needed to confirm the proposed benefits and impact of BCMC on treatment response and patient outcomes.

Keywords: Bladder cancer, quality improvement, neoadjuvant therapy, cystectomy, retrospective study

\section{INTRODUCTION}

Multidisciplinary management of cancer has been widely accepted as a powerful decision-making tool in complex clinical cases [1]. Malignant disease management can involve several different specialties and critical decisions should ideally be made after collaborative discussion among health-care professionals. This has led to the development of various multidisciplinary clinic models, where patients are evaluated by a range of specialists, cases are discussed in the multidisciplinary team meeting and a diagnostic/therapeutic plan is formulated [2]. This multidisciplinary model has been linked to fewer unnecessary delays from diagnosis to treatment initiation, enhanced communication among team members, increased diagnostic accuracy, guided by expert radiology and pathology review, and adherence to national guidelines, as well as improved patient satisfaction scores [2]. Consequently, it has been successfully integrated in the management of a variety of tumor types, such as breast [3], lung cancer [4] and multiple myeloma [5].

Multidisciplinary clinics for genitourinary malignancies have emerged lately, predominantly around prostate cancer care $[6,7]$ and more recently for bladder cancer (BC) care [8-10]. It has been suggested that multidisciplinary clinics are more likely to follow evidence-based practice recommendations compared to health care facilities without this integrative model [2]. For BC in particular, an increase in administration of standard-of-care neoadjuvant chemotherapy (NAC) from $7.7 \%$ to $47.6 \%$ prior to radical cystectomy (RC) has been reported in a tertiary center, after the initiation of a bladder cancer multidisciplinary clinic (BCMC) [8], and referral to high volume tertiary institutions has been encouraged for BC care [11]. In addition, Kulkarni et al. reported a significant change in BC staging after imaging and pathology review of outside records, in combination with additional imaging ordered during multidisciplinary evaluation, dictating vital treatment alterations [10]. In a similar fashion, we hypothesized that a BCMC model at our institution along with the integration of real-time expert central pathology and radiology review would result in significant changes in staging and subsequent treatment recommendations.

\section{MATERIALS AND METHODS}

BCMC is the result of a combined effort of professionals across disciplines of care, focused on patients with urinary tract cancers, including cancers of the bladder, urethra, and upper urinary tract (renal pelvis, ureter). This includes research and clinic care coordinators, nurse practitioners, nurses and ostomy nurses, as well as specialists from medical oncology, radiation oncology, urology, pathology and radiology. Our referral base is broad and comprised mostly of community oncology practices, affiliated and network hospitals and sites, academic and private practice cancer centers inside and outside Washington State. We have adopted the format of a fully integrated clinic, with up to four patients seen on the same (half) day by three different specialists (urology, medical oncology, radiation oncology). During the first hour, an initial history and physical exam are taken by one of the team members (including resident or fellow). During the second hour, in a multidisciplinary conference, the cases are presented to the group. Radiology and pathology images are reviewed by subspecialty radiology and pathology experts. A concrete treatment recommendation plan with relevant options is determined, including consideration for standard-ofcare therapies and appropriate clinical trials. For the subsequent two hours, each service (urology, radiation oncology and medical oncology) will meet with the patient, outlining treatment recommendations (Fig. 1). Patients who are candidates for surgery are seen by the stoma nursing team and referrals are placed to physical therapy, psychology/psychiatry, social work/case manager, nutrition, financial services and palliative care, as necessary.

After obtaining institutional review board (IRB study 00001683) approval, we retrospectively identified all consecutive patients with $\mathrm{BC}$ referred from outside providers to BCMC, from January 2014 to December 2017. In compliance with the IRB 


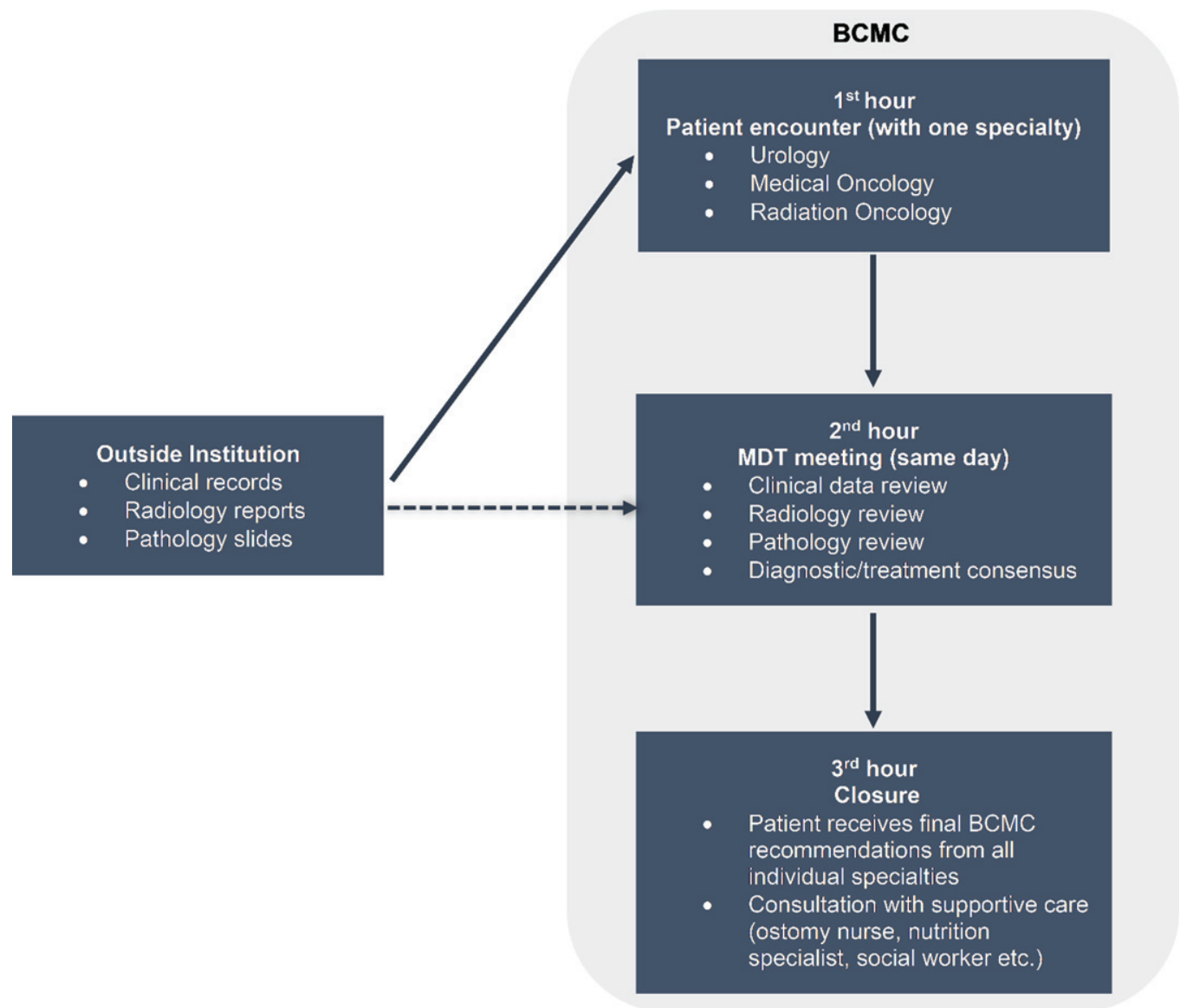

Fig. 1. Bladder Cancer Multidisciplinary Clinic format. Abbreviations: BCMC - bladder cancer multidisciplinary clinic, MDT - multidisciplinary team.

regulations we obtained waiver of consent, also considering the retrospective and observational nature of the study. Baseline clinical characteristics, diagnosis, imaging and pathology data from outside providers and our institution were extracted. Internal referrals and patients with inadequate data were excluded. Then, we retrospectively assessed the concordance between outside/referral imaging \& pathology data and BCMC review with the aim to identify changes in interpretation and whether these changes dictated alternative management plans. As all referrals were evaluated prior to definitive therapy, clinical staging was determined by a combination of clinical, imaging and pathologic data from transurethral resection of bladder tumors (TURBTs). American Joint Committee on Cancer (AJCC) 2017 staging system was applied. Descriptive statistics and chi-squared tests were used for the analyses.

\section{RESULTS}

\section{A. Baseline clinicopathologic characteristics}

We identified 233 patients who were (externally) referred between 2014-2017, with complete radiographic and pathology data available for 201 patients. Data are summarized in Table 1.

\section{B. Pathology and radiology review}

After initial pathology and radiology review, changes in 91 (45\%) patients were noted: $38(19 \%)$ only in histology, $32(16 \%)$ only in imaging reports and $21(10 \%)$ in both. Overall, pathology interpretation changes were made in 59 (29\%) patients (Fig. 2); $43(21 \%)$ were due to histologic variant (including $\%$ ) addition/change, 9 (4.5\%) had lymphovascular 
Table 1

Baseline clinicopathologic data at BCMC referra

\begin{tabular}{|c|c|}
\hline Demographics & $\mathrm{N}=201$ \\
\hline Median Age (IQR) & $69(60-75)$ \\
\hline Males, $n(\%)$ & $150(75)$ \\
\hline Females, $n(\%)$ & $51(25)$ \\
\hline \multicolumn{2}{|l|}{ ECOG Performance Status } \\
\hline $0-1, n(\%)$ & $167(83)$ \\
\hline $2+, n(\%)$ & $32(16)$ \\
\hline N/A & $2(1)$ \\
\hline \multicolumn{2}{|l|}{ Tumor Site } \\
\hline Bladder, $n(\%)$ & $194(96.5)$ \\
\hline UTUC, $n(\%)$ & $3(1.5)$ \\
\hline Urethra, $n(\%)$ & $4(2)$ \\
\hline \multicolumn{2}{|l|}{ Tumor Size } \\
\hline$<2 \mathrm{~cm}, n(\%)$ & $30(15)$ \\
\hline $2-5 \mathrm{~cm}, n(\%)$ & $80(40)$ \\
\hline$>5 \mathrm{~cm}, n(\%)$ & $91(45)$ \\
\hline \multicolumn{2}{|l|}{ Hydronephrosis } \\
\hline Unilateral, $n(\%)$ & $46(23)$ \\
\hline Bilateral, $n(\%)$ & $16(8)$ \\
\hline \multicolumn{2}{|l|}{ Clinical stage at referral } \\
\hline$<\mathrm{T} 2$ & $27(13)$ \\
\hline $\mathrm{T} 2$ & $116(58)$ \\
\hline $\mathrm{T} 3-\mathrm{T} 4$ & $58(29)$ \\
\hline $\mathrm{N}+$ & $15(7.5)$ \\
\hline $\mathrm{M}+$ & $11(5.5)$ \\
\hline \multicolumn{2}{|c|}{$\begin{array}{l}\text { Predominant histologic } \\
\text { subtype (after BCMC review) }\end{array}$} \\
\hline $\mathrm{UC}, n(\%)$ & $181(90)$ \\
\hline Squamous, $n(\%)$ & $12(6)$ \\
\hline $\mathrm{ADCA}, n(\%)$ & $6(3)$ \\
\hline $\mathrm{SCBC}, n(\%)$ & $2(1)$ \\
\hline \multicolumn{2}{|c|}{ Minor component Histologies } \\
\hline Squamous, $n(\%)$ & $26(13)$ \\
\hline Glandular, $n(\%)$ & $18(9)$ \\
\hline Micropapillary, $n(\%)$ & $15(7.5)$ \\
\hline Plasmacytoid, $n(\%)$ & $9(4.5)$ \\
\hline Sarcomatoid, $n(\%)$ & $5(2.5)$ \\
\hline Other, $n(\%)$ & $7(3.5)$ \\
\hline CIS at TURBT & $64(32)$ \\
\hline
\end{tabular}

Abbreviations: BCMC - bladder cancer multidisciplinary clinic, IQR - interquartile range, ECOG Eastern Co-operative Oncology Group, N/A - not available, UTUC - upper tract urothelial carcinoma, T - tumor stage, $\mathrm{N}$ - nodal stage, $\mathrm{M}$ - distant metastasis, UC - urothelial carcinoma, ADCA - adenocarcinoma, SCBC - small cell bladder cancer, CIS - carcinoma in situ, TURBT - transurethral resection of bladder tumor.

invasion (LVI) and/or carcinoma in situ (CIS) status change (from negative to positive) and 2 (1\%) were upgraded from low to high-grade. In terms of staging, $7(3.5 \%)$ of cases were downstaged (pT2 to pT1) and $5(2.5 \%)$ were upstaged (pT1 to pT2). Imaging interpretation changes occurred in $53(26 \%)$ patients, with 26 cases (13\%) upstaged based on local tumor stage classification, 20 (10\%) upstaged based on nodal status and $8(4 \%)$ identified as having distant metastases (Fig. 2). Certain patients underwent a combination of
T, $\mathrm{N}$ or $\mathrm{M}$ status changes. Further diagnostic workup was recommended in 85 patients (42\%) with the most common being additional imaging (computed tomography - CT or magnetic resonance imaging MRI) in 46 (54\%) and positron emission tomography (PET-CT) in 20 (24\%) of those patients. Additionally, repeat TURBT for $15(18 \%)$ and biopsy of metastatic sites for $5(6 \%)$ patients were recommended. By implementing the procedures and imaging noted, we discovered upstaging of 25 additional cases, including tumor ( 5 cases), nodal ( 7 cases) and metastatic site (13 cases) upstaging. Thus, after the completion of BCMC evaluation, $100(50 \%)$ patients had an imaging/pathology change; 41 (20\%) imaging-only modification, $36(18 \%)$ pathologic re-interpretation, while $23(11 \%)$ had combined radiologic/pathologic changes. In terms of clinical staging, 27 (13\%) patients were classified as <T2N0M0, $74(37 \%)$ T2N0M0, 41 (20\%) T3N0M0, 6 (3\%) T4NOM0, 22 (11\%) Tany/N+/M0 and $31(15 \%) \mathrm{M}+$. This represented clinical upstaging (AJCC) in 49 patients (24\%) and downstaging in 7 (3.5\%) patients (Figs. 2, 3).

\section{Treatment recommendations}

Treatment recommendations offered by outside institutions and BCMC are summarized in Table 2. We compared the recommended treatment by the referring team with the recommendations of BCMC (Fig. 4). No outside recommendation was made for $31(15 \%)$ cases. Treatment recommendations were similar in $53(26 \%)$ cases. BCMC treatment plan differed from the referring health care provider plan in $117(58 \%)$ patients. Of those referred for RC only $(n=46), 8(17 \%)$ retained the plan of RC, while $19(41 \%)$ were recommended by BCMC to receive NAC prior to RC, 9 (20\%) switched to palliative chemotherapy, $6(13 \%)$ to chemoradiation and $4(9 \%)$ to intravesical treatment. Out of 64 patients referred for NAC and RC, 38 (59\%) retained their plan, 9 $(14 \%)$ were switched to palliative chemotherapy, 8 (13\%) to RC only, 5 (8\%) to chemoradiation, 2 (3\%) to hospice and $2(3 \%)$ to intravesical therapy. In the group of patients who were referred for chemoradiation $(n=11), 5(45 \%)$ switched to RC, $5(45 \%)$ to NAC and RC and $1(9 \%)$ to hospice. Lastly, out of 6 patients referred for chemotherapy only, 3 (50\%) had no change in treatment, $2(33 \%)$ were transferred to hospice and $1(17 \%)$ switched to NAC and RC. Overall, 184 (91.5\%) patients pursued the treatment plan proposed by BCMC. A total of 115 patients $(57 \%)$ received the entire bladder cancer treatment 


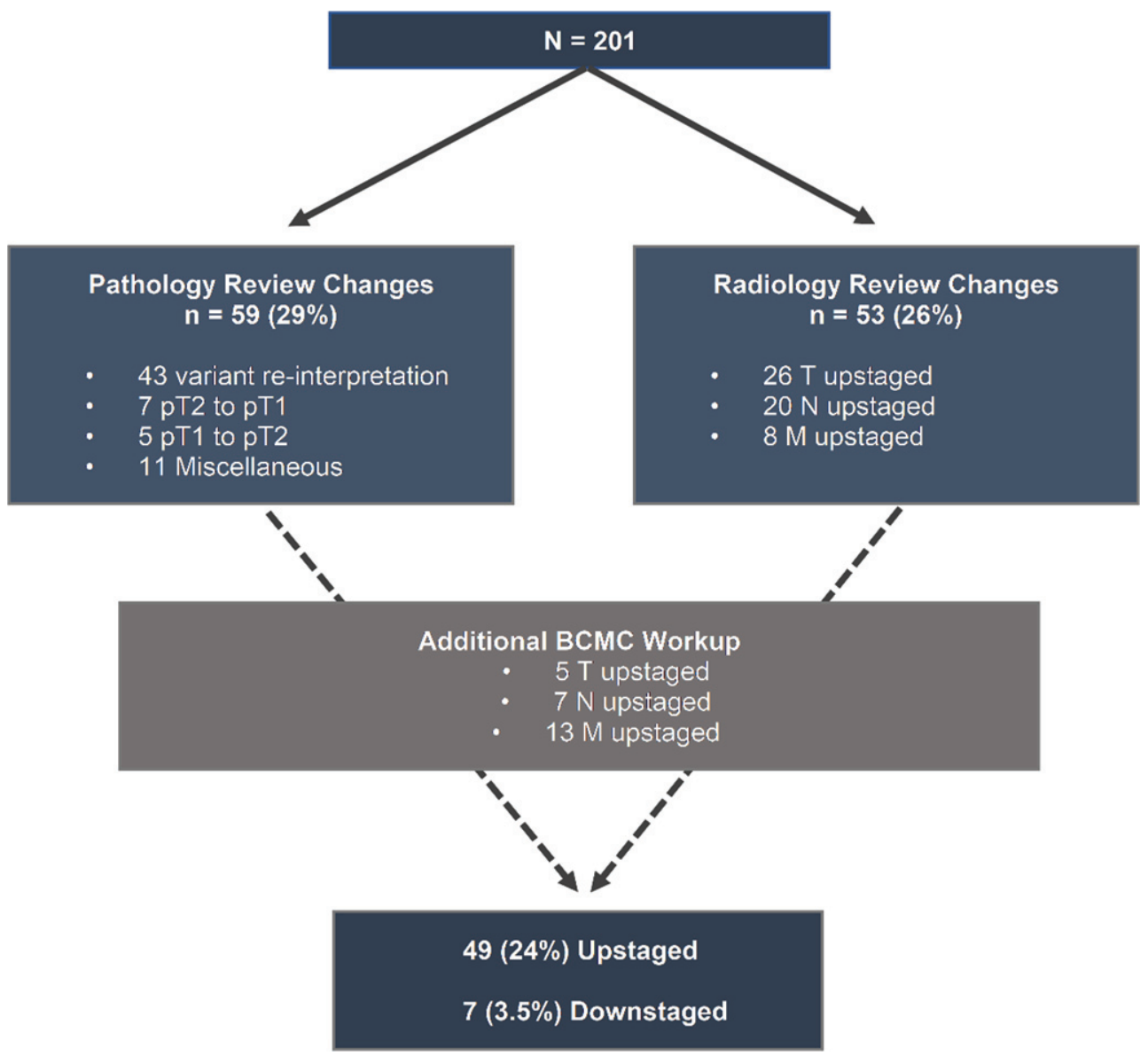

Fig. 2. Diagnostic changes during radiology/pathology review and additional workup. Abbreviations: BCMC - bladder cancer multidisciplinary clinic, T-tumor stage, N-nodal stage, M-distant metastasis, $\mathrm{p}$ - pathologic.

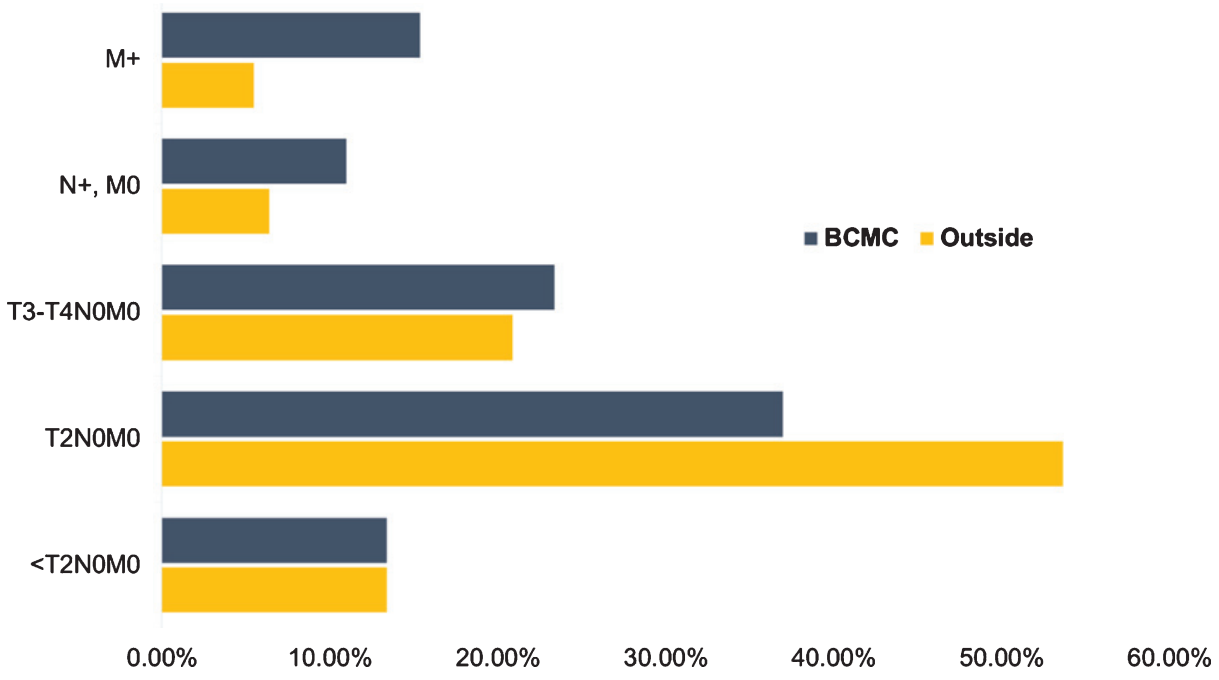

Fig. 3. Clinical staging differences between outside institutions and Bladder Cancer Multidisciplinary Clinic. Abbreviations: BCMC Bladder Cancer Multidisciplinary Clinic, $\mathrm{T}$ - tumor stage, $\mathrm{N}$ - nodal stage, $\mathrm{M}$ - distant metastasis. 
at our institution and 69 (34\%) were treated locally, whereas in 17 (8.5\%) cases, more than one institution was involved in management (e.g. NAC administration outside, followed by RC in our institution). In addition, based on BCMC, 11 patients were enrolled to clinical trials, 9 in the neoadjuvant setting and 2 in the metastatic.

In the subgroup with modified treatment $(n=117)$, $67(57 \%)$ had imaging and/or pathology changes: $23(20 \%)$ pathology-related, 28 (24\%) radiologyrelated and $16(14 \%)$ combined. Treatment plan was modified due to those changes in $43(37 \%)$ patients: new imaging findings influenced $29(25 \%)$, $9(8 \%)$ were modified due to pathology, $5(4 \%)$ due to imaging/pathology combined. In 11/34 (32\%) patients with imaging-directed modification, this happened due to disease progression identified during additional workup. In terms of pathology-dictated treatment alteration, alone or combined with radiology $(n=14)$, this was due to restaging (9 patients) or variant modification (5 patients). In the subset of patients without treatment modifications $(n=53), 18$ (34\%) changes were noted; 8 (15\%) had pathology only changes, $6(11 \%)$ image only changes and 4 (8\%) had both. Using chi-squared test, patients with radiology changes had a higher proportion of treatment plan modifications, compared to those without such changes $(p=0.008)$. Histology re-interpretation showed a non-significant trend towards association with treatment modification $(p=0.08)$. The rest of treatment modifications $(63 \%)$ were due to expert opinion, based on the background of standard-of-
Table 2

Staging and treatment differences between multidisciplinary clinic and referring institutions

\begin{tabular}{lcc}
\hline & Outside, $n(\%)$ & BCMC, $n(\%$, diff $)$ \\
\hline Clinical staging & & \\
<T2N0M0 & $27(13)$ & $27(13,0)$ \\
T2N0M0 & $108(54)$ & $74(37,-17)$ \\
T3N0M0 & $38(19)$ & $41(20,+1)$ \\
T4N0M0 & $4(2)$ & $6(3,+1)$ \\
N+, M0 & $13(6.5)$ & $22(11,+4.5)$ \\
M+ & $11(5.5)$ & $31(15,+9.5)$ \\
\hline Upstagings & & \\
T & & $33(16)$ \\
N & & $27(13)$ \\
M & & $21(10)$ \\
TNM combined & & $49(24)$ \\
\hline Downstagings & & $6(3)$ \\
T & & $1(0.5)$ \\
M & & $7(3.5)$ \\
TNM combined & & \\
Treatment plan & & $34(17,-6)$ \\
RC only & $46(23)$ & $94(47,+15)$ \\
RC and NAC & $64(32)$ & $21(10,+4.5)$ \\
CCRT & $11(5.5)$ & $30(15,+12)$ \\
Chemotherapy only & $6(3)$ & $22(11,-10)$ \\
Other & $43(21)$ & 0 \\
Missing & $31(15)$ & \\
\hline
\end{tabular}

Abbreviations: BCMC - bladder cancer multidisciplinary clinic, diff - \% difference, $\mathrm{T}$ - tumor stage, $\mathrm{N}$ - nodal stage, $\mathrm{M}$ - distant metastasis, RC - radical cystectomy, NAC - neoadjuvant chemotherapy, CCRT - concurrent chemoradiation therapy.

care recommendations, while certain patients elected alternative treatment modalities, when they were provided with the option (e.g. bladder preserving therapy instead of radical cystectomy).

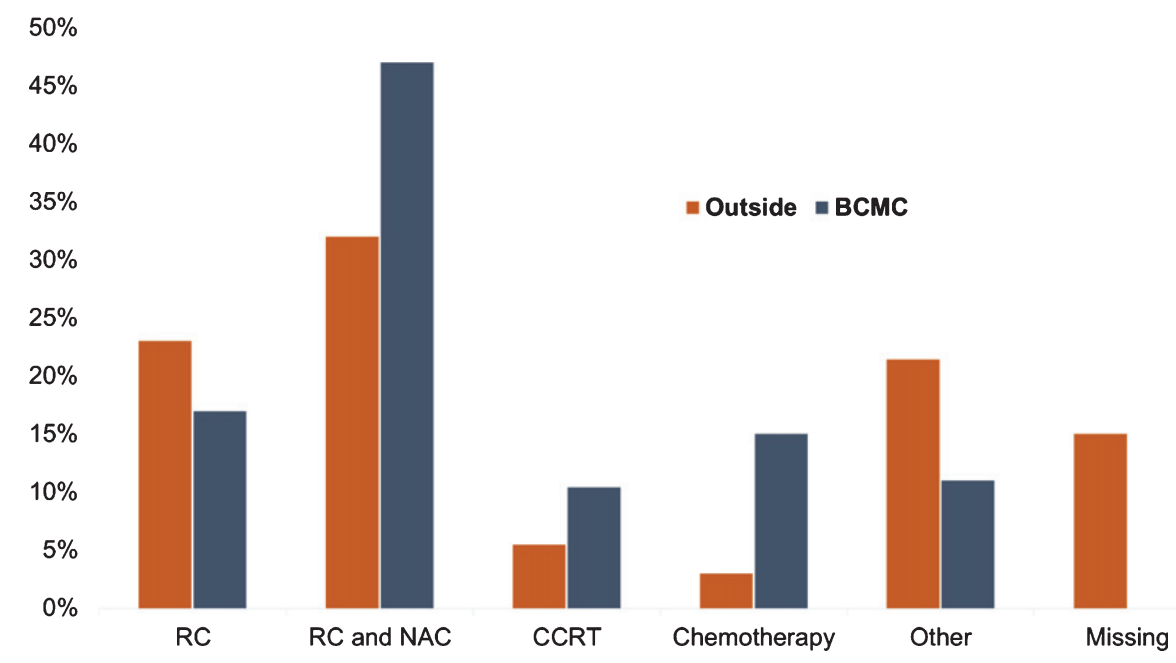

Fig. 4. Differences in recommended treatment plan between outside institutions and Bladder Cancer Multidisciplinary Clinic. Abbreviations: BCMC - bladder cancer multidisciplinary clinic, RC - radical cystectomy, NAC - neoadjuvant chemotherapy, CCRT - concurrent chemoradiation therapy. 


\section{DISCUSSION}

The initiation of a multispecialty bladder cancer clinic at our institution led to changes in staging, pathologic interpretation and treatment recommendations for patients evaluated, highlighting the importance of this comprehensive approach for patient care. The combination of imaging/pathology review and BCMC-ordered diagnostic workup revealed changes in $50 \%$ of the patients, almost equally attributed to histology and radiology. Notably, radiologic changes were always related to modifications in $\mathrm{T}, \mathrm{N}$ or $\mathrm{M}$ staging, whereas most histologic changes were associated with variant re-interpretation, with a few cases leading to alterations in pathologic stage. These findings led to clinical restaging in $28 \%$ of referred patients and often influenced treatment decisions. Overall, 58\% of patients had their initial therapeutic plan modified. BCMC more frequently offered NAC followed by $\mathrm{RC}$, compared to outside providers, as most patients who were referred with an RC-only recommendation had preoperative chemotherapy added to their plan. Chemoradiation was also an option that was frequently provided in our clinic, almost twice as often compared to referring institutions. In converse, none of the 11 patients referred for chemoradiation had the same recommendation by our clinic, however, other cases were deemed appropriate for this approach. There are certain parameters that may render a patient an ideal candidate for chemoradiation for bladder preservation, which were not met in those 11 cases. There is a broader opportunity for the bladder cancer community to discuss updated criteria for trimodality therapy and raise awareness of bladder preservation. Clinical trials, such as SN1806 (NCT03775265), represent an excellent option for patients who meet eligibility criteria. In addition, a proportion of patients who were referred as surgical candidates (with or without NAC), were found ineligible for definitive treatment in BCMC and were switched to a palliative chemotherapy plan. These modifications in treatment were guided by radiology/histology findings during BCMC evaluation in $37 \%$ of the cases, but only radiologic changes had a statistically significant association with changes in management. This could be explained by the different nature of changes between histology and radiology (variant changes vs changes in stage). BCMC recommendations were widely accepted by patients (>90\%) and they frequently $(66 \%)$ elected our institution for all or part of their care. Crite- ria and algorithms for treatment recommendations were based on NCCN, AUA and other published guidelines, local care pathways, and expert consensus opinion. In a number of cases, more than one option was deemed appropriate and those options were discussed with the patient in detail by the providers. It is critical that patients make an informed/shared decision after being made aware of the totality of options (both standard of care and relevant clinical trials) in an unbiased, careful, well-balanced and evidence-based manner.

Reports on BCMC models and their impact on patient care are limited in the literature. Nayan et al. documented an increase in NAC utilization after a BCMC model was adopted in their institution, from $8 \%$ to $48 \%$, although a similar trend was also observed across the years, but to a lesser extent, in patients that did not attend their multidisciplinary clinic [8]. However, there was no data comparison with outside referring providers in that study. Kulkarni et al. reported a change in tumor stage in $36 \%$ of patients referred to their BCMC, after imaging/pathology review and additional diagnostic workup were performed, with $11 \%$ attributed to pathology [10]. There was a change in treatment recommendations in $33 \%$ of patients, derived from their BCMC experience, and the most common therapies that they offered were RC (30\%) and chemoradiation $(32 \%)$; NAC was administered in $20 \%$ of patients who underwent RC. Other treatment modalities included palliative care, intravesical chemotherapy and cystoscopy surveillance [10]. Due to the paucity of literature involving BCMC models, comparisons with other institutional experiences remains limited.

Interestingly, there are parallels derived from the multidisciplinary care of other malignancies. In a breast cancer multidisciplinary clinic, imaging review led to interpretation changes in $45 \%$ of patients and pathology review in $29 \%$ of patients, dictating modifications in surgical management in $20 \%$ of patients [3]. Also, in a multiple myeloma multidisciplinary setting, imaging review by an expert radiologist revealed $23 \%$ additional bone abnormalities, not previously reported [5]. Lastly, $19 \%$ of patients with pancreatic cancer underwent clinical restaging and $24 \%$ received a change in treatment recommendations based on a pancreatic cancer multidisciplinary model of care [12]. Our findings, along with the literature data, support the need for imaging studies to be reviewed by radiologists with relevant expertise, if possible. 
Although ideal, not all patients and settings have the resources for a multi-specialty clinic model due to financial, administrative and several other logistical factors. At present, only four patients are seen per half-day clinic, due to capacity limitations. The ability to increase patient access requires efforts to increase clinic efficiency, including hiring more staff, as well as optimizing clinical operations (e.g. intake and scheduling), expedited review of cases by experts and coordinating with other disciplines (radiology, pathology, genetics), which may provide more space and allocated time. In fact, those quality improvement initiatives are actively being explored at our center. Another critical factor is the development of criteria for patients most appropriate for multidisciplinary care referral. Those criteria can be the focused objective of discussions among experts, whereas the Bladder Cancer Advocacy Network (BCAN) Think Tank Meeting may serve as a relevant forum for such detailed consideration. Despite the limitations, lessons can be learned from the example of our BCMC clinic. First, accurate imaging and pathology interpretation is of vital importance in order to develop a personalized patient-centered management plan. Second, optimal and dynamic communication between the specialties that participate in $\mathrm{BC}$ care is essential for accurate staging and the complex decision-making that $\mathrm{BC}$ requires. Patient access to care, transportation, lodging, parking, health literacy, expectations, beliefs and perceptions can all complicate the provision of expert multi-disciplinary care. Further discussion among various stakeholders is necessary to overcome these notable obstacles. Improved utilization of telehealth with appropriate resource allocation and infrastructure may be one such opportunity for increasing access for patients as well as providers for consultation with specific tumor type experts. There are already several applications of telemedicine in the field of oncology, known as tele-oncology [13]. With the development of software for remote video calls and teleconferences, patients could receive a real-time evaluation by a multidisciplinary team. Telepathology and teleradiology would be particularly important in that setting by allowing specialists to remotely access radiographic and pathology images uploaded to a cloud service and provide a diagnostic outline without the need for physical presence. This could even be extended in providing eligibility assessment and, potentially, consent for participation to clinical trials (depending on regulatory approval), among other management options [13]. Moreover, patient advocacy groups, such as BCAN, could potentially be involved in facilitating community outreach to numerous sites, raise awareness about expert providers and legislative authorities, and help promote the use of telehealth as an efficient and less costly means of patient care. Taking into consideration that a number of patients with bladder cancer may not necessarily require multidisciplinary care, the development of a 'consensus' regarding criteria that would warrantee patient referral could be undertaken. In that regard, BCAN, along with other advocacy groups and cancer societies, could help setting the conceptual framework for those discussions. In that way, optimal utilization of multidisciplinary care can be regulated by an evidence-based model and facilitate access to remote areas and patients.

Limitations of our study include the retrospective nature, with potential selection and confounding biases, single institutional experience, lack of followup, treatment response, patient satisfaction scores and outcomes data. Also, all the patients described here were from external referrals with incomplete data regarding initial diagnosis and baseline clinicopathologic characteristics in certain cases. Furthermore, in a third of patients with radiology changes, treatment was altered due to disease progression identified during BCMC workup. It is possible that these patients would have received similar treatment recommendations from the referring provider, should restaging had been performed by those institutions. In general, most patients were seen by our clinic within 2-3 weeks from the initial referral. Potential delay in the referral process may possibly account for interim disease progression; therefore, expedited clinic review of cases is ideal. However, it should be emphasized that BCMC evaluation was not only made on the grounds of a "second opinion". On the contrary, definitive therapy for those patients was scheduled to occur through BCMC, while restaging was typically performed in the usual timeframe between diagnosis and local therapy and reflected concerns that distant disease was not adequately considered or concerns regarding interval aggressiveness of disease. Another limitation of the study, and an aspect that would be interesting to investigate in the future, is that the number of patients who enrolled on clinical trials was relatively low due to trial availability at that particular time period. Future studies should also evaluate the impact of BCMC on clinical outcomes, as well as data on cost-effectiveness, healthcare utilization, treatment complications, evidence and value-based care. 


\section{CONCLUSIONS}

Outside referrals to our BCMC experienced significant changes in imaging and/or histology interpretation, while modified treatment recommendations were made in $58 \%$ of referred patients, with increased use of neoadjuvant chemotherapy, bladder preservation, and palliative care. These findings highlight the fact that multidisciplinary care in BC is still under development and more studies are needed to evaluate the importance of this health-care model in patient satisfaction and clinical outcomes.

\section{AUTHOR CONTRIBUTIONS}

Reflecting the study nature, all authors contributed in the conception, performance and interpretation of data presented, as well as in the manuscript preparation and approval of the final version.

\section{ACKNOWLEDGMENTS}

We would like to thank the patients and families who have been part of the BCMC and provided feedback for improvement of the program, all our clinic coordinators and staff, as well as referring providers.

\section{FUNDING}

Parts of this study were funded by Howard J Cohen Bladder Cancer Foundation.

\section{CONFLICT OF INTEREST}

Leonidas N. Diamantopoulos, Brian R. Winters, Steven D. Ngo, Jing Zeng, Jay Liao, Jean H. Lee, Joanna Daya, Maria S. Tretiakova, Funda VakarLopez, have no conflicts to disclose.

The following conflicts of interest, unrelated to this manuscript, are reported by the co-authors:

\section{Petros Grivas}

Consulting (last 3 years): AstraZeneca; Bayer; Biocept; Bristol-Myers Squibb; Clovis Oncology; Dendreon; Driver; EMD Serono; Exelixis; Foundation Medicine; Genentech; Heron Therapeutics; Janssen; Merck \& Co.; Mirati Therapeutics; Pfizer; Seattle Genetics; QED Therapeutics, GlaxoSmithKline, Roche.
Educational program (not current, with direct input in slide content): Bristol-Myers Squibb; Genentech.

Research Funding to Institution: AstraZeneca, Bayer; Genentech/Roche; Merck; Mirati Therapeutics; Oncogenex; Pfizer, Clovis Oncology, Bavarian Nordic, Immunomedics, Debiopharm, Bristol-Myers Squibb.

\section{Andrew C. Hsieh}

Honoraria - Hotspot Therapeutics

Research Funding - eFFECTOR Inc

Patents, Royalties, Other Intellectual Property MTOR modulators and uses thereof Patent number: 9629843; USE OF TRANSLATIONAL PROFILING TO IDENTIFY TARGET MOLECULES FOR

THERAPEUTIC TREATMENT Publication number: 20140288097

\section{George R. Schade}

Patents, Royalties, Other Intellectual Property Global Cancer Technology

\section{Heather H. Cheng}

Research Funding - Astellas Medivation; Clovis Oncology; Color Foundation;

Inovio Pharmaceuticals; Janssen; Sanofi

Sarah P. Psutka

Honoraria - Prime Education

Travel, Accommodations, Expenses - Prime Education

\section{Robert B. Montgomery}

Research Funding - AstraZeneca; ESSA; Ferring; Janssen Oncology

\section{Evan Y. Yu}

Consulting - Amgen, AstraZeneca, Bayer, Dendreon, Janssen, Merck, Pharmacyclics, Seattle Genetics. Research Grant Funding to University of Washington - Dendreon, Merck, Seattle Genetics

\section{Michael T. Schweizer}

Paid consultant/advisor to Janssen.

Research Grant funding to University of Washington from Janssen, AstraZeneca, Zenith, Pfizer and Hoffmann-La Roche 


\section{John L. Gore}

Research Grant funding from Ferring Pharmaceuticals

\section{Manjiri Dighe}

Research Grant from Philips Medical Imaging

\section{Daniel W. Lin}

DSMB for the POTOMAC study with AstraZeneca Consulting or Advisory Role - Astellas Pharma; Clovis Oncology; Dendreon

Research Funding - GenomeDx; Genomic Health; MDxHealth

\section{Lawrence D. True}

Stock and Other Ownership Interests - Lightspeed Micro Research Funding - Ventana Medical Systems

Patents, Royalties, Other Intellectual Property Lens on an open-top lightsheet microscope

\section{Kenneth J. Russell}

\section{Medical/Scientific Consultant for Varian Corpora-} tion.

\section{Jonathan L. Wright}

Royalties - UpToDate.

Clinical Trials - Movember Foundation, Merck, Nucleix, Altor Biosciences.

Consulting - Sanofi Genzyme

\section{REFERENCES}

[1] Soukup T, Lamb BW, Arora S, Darzi A, Sevdalis N, Green JS. Successful strategies in implementing a multidisciplinary team working in the care of patients with cancer: An overview and synthesis of the available literature. Journal of multidisciplinary healthcare. 2018;11:49-61.

[2] Horvath LE, Yordan E, Malhotra D, Leyva I, Bortel K, Schalk D, Mellinger P, Huml M, Kesslering C, Huml J. Multidisciplinary care in the oncology setting: Historical perspective and data from lung and gynecology multidisciplinary clinics. Journal of Oncology Practice. 2010;6(6):e21-e6.

[3] Newman EA, Guest AB, Helvie MA, Roubidoux MA, Chang AE, Kleer CG, Diehl KM, Cimmino VM, Pierce
L, Hayes D, Newman LA, Sabel MS. Changes in surgical management resulting from case review at a breast cancer multidisciplinary tumor board. Cancer. 2006;107(10):234651.

[4] Conron M, Phuah S, Steinfort D, Dabscheck E, Wright G, Hart D. Analysis of multidisciplinary lung cancer practice. Internal Medicine Journal. 2007;37(1):18-25.

[5] Singh J, Fairbairn KJ, Williams C, Das-Gupta EP, Russell NH, Byrne JL. Expert radiological review of skeletal surveys identifies additional abnormalities in $23 \%$ of cases: Further evidence for the value of myeloma multidisciplinary teams in the accurate staging and treatment of myeloma patients. British Journal of Haematology. 2007;137(2):172-3

[6] Aizer AA, Paly JJ, Efstathiou JA. Multidisciplinary care and management selection in prostate cancer. Seminars in Radiation Oncology. 2013;23(3):157-64.

[7] Loblaw A, Barkin J, Buckley R, Chung H, Kell J, Singal R, Spodek J, Vesprini D, Flax S. Improving prostate cancer care collaboratively - a multidisciplinary, formal, consensus-based approach. The Canadian Journal of Urology. 2017;24(1):8646-50.

[8] Nayan M, Bhindi B, Yu JL, Mamdani M, Fleshner NE, Hermanns T, Chung P, Milosevic M, Bristow R, Warde P, Hamilton RJ, Finelli A, Jewett MA, Zlotta AR, Sridhar SS, Kulkarni GS. The initiation of a multidisciplinary bladder cancer clinic and the uptake of neoadjuvant chemotherapy: A time-series analysis. Canadian Urological Association journal=Journal de l'Association des urologues du Canada. 2016;10(1-2):25-30.

[9] Harshman LC, Tripathi A, Kaag M, Efstathiou JA, Apolo AB, Hoffman-Censits JH, Stadler WM, Yu EY, Bochner BH, Skinner EC, Downs T, Kiltie AE, Bajorin DF, Guru K, Shipley WU, Steinberg GD, Hahn NM, Sridhar SS. Contemporary patterns of multidisciplinary care in patients with muscle-invasive bladder cancer. Clinical Genitourinary Cancer. 2018;16(3):213-8.

[10] Kulkarni GS, Hermanns T, Wei Y, Bhindi B, Satkunasivam R, Athanasopoulos P, Bostrom PJ, Kuk C, Li K, Templeton AJ, Sridhar SS, van der Kwast TH, Chung P, Bristow RG, Milosevic M, Warde P, Fleshner NE, Jewett MAS, Bashir S, Zlotta AR. Propensity score analysis of radical cystectomy versus bladder-sparing trimodal therapy in the setting of a multidisciplinary bladder cancer clinic. Journal of Clinical Oncology: Official Journal of the American Society of Clinical Oncology. 2017;35(20):2299-305.

[11] Koshkin VS, Grivas P. Perioperative chemotherapy for muscle-invasive bladder cancer: The importance of multidisciplinary management for evidence-based practice and transformative research. Translational Andrology and Urology. 2018;7(3):504-7.

[12] Pawlik TM, Laheru D, Hruban RH, Coleman J, Wolfgang CL, Campbell K, Ali S, Fishman EK, Schulick RD, Herman JM. Evaluating the impact of a single-day multidisciplinary clinic on the management of pancreatic cancer. Annals of Surgical Oncology. 2008;15(8):2081-8.

[13] Sirintrapun SJ, Lopez AM. Telemedicine in cancer care. American Society of Clinical Oncology Educational book American Society of Clinical Oncology Annual Meeting. 2018;38:540-5. 